\title{
SOME ALGEBRAIC SETS OF HIGH LOCAL COHOMOLOGICAL DIMENSION IN PROJECTIVE SPACE
}

\author{
GENNADY LYUBEZNIK
}

Abstract. Let $V_{0}, \ldots, V_{[n / t]}$ be algebraic sets of pure codimension $t$ in $P^{n}$, and suppose $\cap V_{i}$ is empty. Then $P^{n}-U V_{i}$ has cohomological dimension $n-[n / t]$.

If $U$ is a scheme, then $\operatorname{cd}(U)$, the cohomological dimension of $U$, is the largest integer $i$ such that there exists a quasi-coherent sheaf $F$ on $U$ such that $H^{i}(F) \neq 0$.

In [1], G. Faltings proved that if $V$ is an algebraic set of pure codimension $t$ in $P^{n}$, then

$$
\operatorname{cd}\left(P^{n}-V\right) \leqslant n-[n / t] .
$$

This note gives some algebraic sets for which equality holds in (1).

Theorem. Put $s=[n / t]$ and let $V=V_{0} \cup V_{1} \cup \cdots \cup V_{s}$ be the union of $s+1$ algebraic sets of pure codimension $t$ in general position in $P^{n}$ (i.e. such that the intersection of all of them is empty). Then

$$
\operatorname{cd}\left(P^{n}-V\right)=n-[n / t] .
$$

This theorem (from the author's thesis [4]) answers the conjecture from [3] in the affirmative and covers all three examples from [3], but not the statement of the main theorem.

For a proof it is convenient to translate the problem into an algebraic language. Put $R_{n}=k\left[x_{0}, \ldots, x_{n}\right]_{\left(x_{0} \ldots, x_{n}\right)}$ and let $\mathfrak{A}$ be the defining ideal of $V$ in $R_{n}$. Then the cohomological dimension of $P^{n}-V$ is the largest integer $i$ such that $H_{\mathscr{U}}^{i+1}\left(R_{n}\right) \neq 0$ (cf. [2]).

Lemma. Put $s=[n / t]$ and let $\mathfrak{H}_{0}, \mathfrak{H}_{1}, \ldots, \mathfrak{A}_{j}$ be $j+1$ homogeneous ideals of pure height $t$ in $R_{n}$. Put $\beta_{j}=\sum_{r=0}^{r=j} \mathfrak{A}_{r}$. Then $H_{\beta_{i}}^{i}\left(R_{n}\right)=0$ if $i \geqslant n-s+j+2$.

Proof. If $j=0$, the result follows from (1). Put $\beta_{j-1}=\sum_{r=0}^{r=j-1} \mathfrak{A}_{r}$. Then $\beta_{j-1} \cap \mathfrak{U}_{j}$ has the same radical as $\gamma_{j-1}=\sum_{r=0}^{r=j-1}\left(\mathfrak{U}_{r} \cap \mathfrak{A}_{j}\right)$. Since $\beta_{j-1}$ and $\gamma_{j-1}$ are sums of $j-1$ ideals of pure heights $t$ in $R_{n}$, we may assume that $H_{\beta_{1},}^{i}\left(R_{n}\right)=H_{\gamma_{1},}^{i}\left(R_{n}\right)=0$ for all $i \geqslant n-s+j+1$. We also know that $H_{\mathfrak{H}_{1}}^{i}\left(R_{n}\right)=0$ if $i \geqslant n-s+2$. The Mayer-Vietoris long exact sequence gives

$$
H_{\gamma_{1}, 1}^{i}\left(R_{n}\right) \rightarrow H_{\beta_{1}}^{i+1}\left(R_{n}\right) \rightarrow H_{\beta_{1}, 1}^{i+1}\left(R_{n}\right) \oplus H_{\mathfrak{H}_{1}}^{i+1}\left(R_{n}\right)
$$

and this proves the Lemma.

Received by the editors April 5, 1984.

1980 Mathematics Subject Classification. Primary 14B15. 13D()3. 
Proof of the Theorem. Let $\mathfrak{U}_{0}, \ldots, \mathfrak{A}_{s}$ be the defining ideals of $V_{0}, \ldots, V_{s}$ in $R_{n}$. Put $\mathfrak{\subseteq}_{j}=\mathfrak{A}_{0} \cap \mathfrak{A}_{1} \cap \cdots \cap \mathfrak{U}_{j}+\mathfrak{A}_{j+1}+\cdots+\mathfrak{U}_{s}$. Then the biggest integer $i$ for which $H_{\Xi_{s}}^{i}\left(R_{n}\right) \neq 0$ is $i=n-j+1$. We are going to prove this by induction on $j$ and the theorem will follow by putting $j=s$.

For $j=0, \Im_{j}$ is $m$-primary, where $m$ is the maximal ideal of $R_{n}$ and the above claim is well known in this case. Assume $j>0$ and assume the Theorem proven for $j-1$. Put $\mathfrak{U}^{\prime}=\mathfrak{U}_{j}+\mathfrak{U}_{j+1}+\cdots+\mathfrak{U}_{s}$ and $\mathfrak{U}^{\prime \prime}=\mathfrak{U}_{0} \cap \mathfrak{U}_{1} \cap \cdots \cap \mathfrak{U}_{j-1}+\mathfrak{U}_{j+1}$ $+\mathfrak{A}_{j+2}+\cdots+\mathfrak{U}_{s}$. Then $\mathfrak{S}_{j}=\mathfrak{U}^{\prime} \cap \mathfrak{U}^{\prime \prime}$ and $\mathfrak{S}_{j-1}=\mathfrak{U}^{\prime}+\mathfrak{U}^{\prime \prime}$. By the Lemma $H_{\mathfrak{Q}}^{i}\left(R_{n}\right)=H_{\mathscr{Q}}^{i}{ }^{\prime \prime}\left(R_{n}\right)=0$ for all $i \geqslant n-j+2$. The claim now follows from the Mayer-Vietoris sequence considering the induction hypothesis. Q.E.D.

REMARK. The above Lemma gives a lower bound on the number of algebraic sets of given codimension which are needed to cut out a given algebraic subset of $\mathbf{P}^{n}$ set-theoretically. Namely, if $V \subset \mathbf{P}^{n}$ and $\operatorname{cd}\left(\mathbf{P}^{n}-V\right)=v$, then we need at least $v+1-(n-[n / t])$ algebraic subsets of pure codimension $t$ to cut out $V$ settheoretically.

Faltings' inequality (1) and the fact that it is exact for all $n$ and $t$ (our Theorem) suggest the following.

Conjecture. Every algebraic subset of $\mathbf{P}^{n}$ of pure codimension $t$ is a set-theoretic intersection of $n+1-[n / t]$ hypersurfaces $[4, \mathrm{p} .8]$.

For additional supporting evidence for this conjecture see [4, Theorem 6].

\section{REFERENCES}

1. G. Faltings, Üher locale Kohomologiegruppen hoher Ordnung, J. Reine Angew. Math. 313 (1980), 43-51.

2. R. Hartshorne, Cohomological dimension of algebraic varieties, Ann. of Math. 88 (1968), 403-450.

3. G. Lyubeznik, On set-theoretic intersections, J. Algebra 87 (1984), 105-112.

4. Set-theoretic intersections and monomial ideals, Thesis, Columbia University, 1984.

Department of Mathematics , Columbia University, New York, New York 10027

Current address: Department of Mathematics, Purdue University, West Lafayette, Indiana 47907 